European Journal of Public Health, Vol. 26, No. 2, 272-276

(C) The Author 2015. Published by Oxford University Press on behalf of the European Public Health Association. All rights reserved. doi:10.1093/eurpub/ckv208 Advance Access published on 16 November 2015

\title{
Effectiveness of an active commuting school-based intervention at 6-month follow-up
}

\author{
Emilio Villa-González ${ }^{1,2}$, Jonatan R. Ruiz², Dianne S. Ward ${ }^{3}$ Palma Chillón ${ }^{2}$ \\ 1 Department of Physical Culture, School of Health Sciences, National University of Chimborazo, Riobamba, Ecuador \\ 2 PROFITH "PROmoting FITness and Health through physical activity" research group, Department of Physical Education \\ and Sport, School of Sport Sciences, University of Granada, Granada, Spain \\ 3 Department of Nutrition, University of North Carolina, Chapel Hill, NC, USA
}

Correspondence: Emilio Villa-González, Department of Physical Culture, School of Health Sciences, National University of Chimborazo, Avda. Antonio José de Sucre, Km. 1 1/2 vía a Guano, Riobamba, Ecuador, Tel: (+593) 983968692, Fax: (+34) 9582443 69, e-mail: evilla@unach.edu.ec

\begin{abstract}
Background: Active commuting to school may provide a significant source of physical activity in youth. Previous school-based intervention studies have shown a positive effect on increasing the frequency of active commuting to school in the short-term. However, how the observed effects are after the intervention remains to be investigated. The objective of the present study was to investigate the effects of a school-based intervention on active commuting to school at 6-month follow-up. Methods: A total of 494 children ages 8-11 years from 5 primary schools were invited to the study. The schools were non-randomly allocated into control or experimental group. The experimental group received a 6-month programme focused on increasing active commuting to school, while the control group received no intervention. Frequency and mode of commuting to school were measured using a questionnaire at pre-intervention, post-intervention and 6-month follow-up. Children with valid data on commuting to school and provided data for sex, age and distance from home were included in this study $(n=206)$. Results: There was a significant difference in the change of number of walk and bike travels per week between groups at 6 -month follow-up (Control-Group $=-0.4 \pm 0.3$; Experimental-Group $=0.6 \pm 0.2$; $p=0.019)$. Regarding the frequency of mode of commuting, only a change in walking to school was significantly different between the groups at 6-month follow-up (Difference Follow-up-Post-intervention) (ControlGroup $=-0.6 \pm 0.3$; Experimental-Group $=0.7 \pm 0.2 ; p=0.004$ ). Conclusions: A 6-month school-based intervention focused on increasing active commuting to school could be effective strategy for increasing the frequency of active commuting to school even beyond the period of intervention.
\end{abstract}

\section{Introduction}

$P^{h}$ ysical inactivity in youth continues to be an international epidemic and a potential contributor to chronic disease in adulthood. ${ }^{1}$ Active commuting to school (defined as the use of active means, mainly walking and bicycling, to and/or from school) can provide a significant source of physical activity for children $^{2}$ and has also been associated with improved cardiovascular health. ${ }^{3}$ Moreover, active commuting to school has been associated with a higher cognitive performance in adolescent girls, ${ }^{4}$ reduced stress in children during school day ${ }^{5}$ and a reduced greenhouse gas emissions in neighbourhood schools. ${ }^{6}$

Despite the benefits of active commuting to school, the frequency of active commuting to school has declined dramatically over the past 30 years in the United States ${ }^{7}$ and in the last decade in Spain. ${ }^{8}$ Intervention efforts are therefore necessary to counter this trend. Initiatives such as Safe Routes to School, ${ }^{9}$ the Walking School Bus, ${ }^{10}$ the Walk to School programme ${ }^{11}$ and the School Travel Plan programme $^{12}$ have been implemented to increase children's walking and bicycling to school with some success. A systematic review ${ }^{13}$ conducted to identify intervention studies on active commuting to school (through January 2010), concluded that more research with higher quality study designs and measures was needed to identify the most successful strategies for increasing the frequency of active commuting to school. Most of the existing school-based intervention studies assessed the frequency of active commuting before (pre-intervention) and after (post-intervention) of the intervention programme. ${ }^{13}$ Few studies have measured maintenance of increased active commuting after a period of time from post-intervention (i.e. follow-up). ${ }^{14,15}$ Of these, at least one study observed an increased frequency of active commuting for at least 2 weeks after the intervention $^{14}$ and another a non-significant intervention effect on children's cycling frequency to school at 5-months follow-up. ${ }^{15}$

Thus, the aim of the current study was to investigate the effects of a school-based intervention on active commuting (i.e. walking and biking) to school at 6-month follow-up, with the hypothesis that children's frequency of active commuting to school would be significantly higher than baseline 6 months following the end of the intervention.

\section{Methods}

\section{Participants}

A total of 494 children aged 8-11 years of age were invited to participate in the baseline study. Participants were recruited from five primary schools in the provinces of Granada (Salobreña, $n=119$; Huétor Vega, $n=80$; Santa Fe, $n=96$; the city of Granada, $n=128$ ) and Jaén (Castillo de Locoubín, $n=46$ ) to participate in an intervention to increase walking and biking to school. From these, 469 children were included at baseline because they had valid data on commuting to school. For the current study, only children who had valid data on commuting to school at the three measurement points (i.e. pre-intervention, post-intervention and 6-month follow-up) and provided data for sex, age and distance from home to school were included $(n=206)$.

\section{Study design}

A total of 117 participants from 3 schools of Salobreña, Huétor Vega and Santa Fe took part in the experimental group (EG), which 
received a 6-month intervention programme focused on increasing the level of active commuting to school. A total of 89 participants from the 2 schools from Granada and Castillo de Locoubín took part in the control group (CG), which kept the normal activity at schools. Experimental schools were politically assigned by the local government (i.e. Diputación de Granada) regarding a previous interest from the municipalities where the schools were located. Control schools were selected for comparison by the researchers based on having similar characteristics (i.e. socio-economic level and location-urban vs. rural) to the experimental schools. The school staff and children did not know the assignment prior to being recruited. All the schools were public and primary education schools within the National Educational System. The outcome measures were taken during school days prior to (pre-intervention), immediately following (post-intervention) and after 6 month (follow-up) of the intervention programme in the months of January, June and November, respectively, of the academic year 2011/2012 in every school. All measurements were taken in the same period and the five localizations belonged to the same region with similar weather conditions.

\section{Ethics approval}

The study was conducted within a public health initiative lead by Diputación de Granada (Área de Medio Ambiente). The purpose of this programme was to promote safe and healthy ways of commuting from home to school. An agreement was signed by each experimental school board (decision-making body of a school), Diputación de Granada and the municipalities. All school boards (i.e. control and experimental), parents and students were informed about the study and agreed to participate and a written consent from parents was obtained. The Medical Ethics Committee of Hospital Virgen de las Nieves (Granada, Spain) approved the study design, study protocols and informed consent procedure (case no. 817).

\section{Intervention}

The intervention focused on increasing the levels of active commuting to and from school among children. Both teachers and research team implemented the intervention programme at each school. The intervention period lasted two school terms (6 months) from January to June of 2012. The intervention was focused mainly among children and weakly among parents. The intervention was based in the conceptual framework of active travel in children proposed by Panter et al., ${ }^{16}$ targeting mainly the individual factors such as children perceptions (e.g. safety perception on the way to school) and children attitudes (e.g. independence or motivation to walk). The intervention targeted weakly other determinants previously described, as the urban form or parental perceptions. ${ }^{17-19}$ Children from the EG participated in 6 monthly activities within the intervention programme (each activity ranged from 60 to 120 min each month) during school hours in addition to their regular Physical Education lessons. The intervention included: (i) introductory activities such as a questionnaire of mode of commuting reported by families (parents or grandparents). The objective of this activity was to know how the families commuted in his/her youth and consider whether currently, there were real barriers to active commuting to school for their children. (ii) Reading a story and performing scenes related to active commuting to school. The objective of this activity was to familiarize the children with active commuting to school and the neighbourhood. (iii) Activity of knowledge about the environmental characteristics around the school. The objective was to know the urban environment (i.e. measuring the size of sidewalks and crosswalks, understanding the traffic signs) in the area surrounding the school, in order to know the main features routes to and/or from school that support or limit the behaviour of walking and cycling to school. (iv) Activity of road safety. The objective was to promote road safety, and analyze the relation between vehicles (i.e. cars and bikes) and pedestrians (i.e. supporting older people to cross crosswalks, interviewing pedestrian and drivers, warnings for inadequate behaviours of the car drivers and cyclists) (v) Activity of behaviours in the street. The objective was to know the appropriate behaviours of pedestrians, vehicles and traffic police (i.e. measuring the time to cross a crosswalk and the vehicle's speed, collaborating with the traffic police) (vi) Activity of traditional games. The objective was to practice traditional games and adapted them to the topic of road safety education and active commuting to school (i.e. playing to cooperation games integrating ethical and social behaviours as if they were citizens and traffic polices). These activities were carried out in the classroom (i to ii) and in the school neighbourhood (iii to vi). Children and teachers participated in all scheduled activities (i to vi), while parents only participated in one activity (i). Children in the CG and EG received the usual Physical Education sessions according to the National Education Program in Spain, i.e. 55 min sessions twice per week.

\section{Mode and frequency of commuting to school}

Participants completed a self-report questionnaire regarding the latest weekly patterns of commuting to and from school (Monday to Friday). The modes of commuting were: walk, bike, car, motorcycle and bus. Walking and biking were categorized as active commuting and travelling by car, motorcycle and bus were categorized as passive commuting. This questionnaire has been proposed as the most appropriate measurement for asking about mode of commuting to school based on results from a review of 158 studies. ${ }^{20}$ Children completed the questionnaire with the help of the teacher and members of the research team. The weekly frequency of active commuting to school was expressed as numbers of active travels per week to and from school (range: 0-10). The distance from home to school was estimated using the Internet program Google Map V.6. ${ }^{21}$ The shortest network path between each student's home address and the school measured in meters was used.

\section{Statistical analysis}

The normality of the active commuting variables was assessed using the test Kolmogorov-Smirnov. The intervention effects on the frequency of active commuting and frequency of the mode of commuting per week were studied by one-way analysis co-variance. The change of both frequency of active commuting and frequency of the mode of commuting at post-intervention (Post-interventionPre-intervention difference) and follow-up (Follow-up-Postintervention difference) were included as dependent variables. The group was included as fixed factor (CG or EG), and sex, age, distance from home to school and both pre-intervention and postintervention values of each dependent variable as covariates, respectively. Analyses were performed using the PASW (v. 20.0 for Windows, Chicago, IL, USA), and the level of significance was set to 0.05 .

\section{Results}

Descriptive characteristics of the frequency of the mode of commuting to school at pre-intervention, post-intervention and 6month follow-up are shown in table 1 . There was no significant difference on the frequency of each mode of commuting used between the control and experimental groups at pre-intervention, post-intervention and 6-month follow-up. There was a significant difference on the change of the frequency of active commuting (number of walk and bike travels per week) between the control and experimental groups at 6-month follow-up [CG $=-0.4 \pm 0.3$; $\mathrm{EG}=0.6 \pm 0.2 ; p=0.019$ ] (figure 1). Descriptive values of the frequency of active commuting at pre-intervention, post-intervention and follow-up are shown in figure 2. There was no significant 
Table 1 Descriptive characteristics of the frequency of the mode of commuting at pre-intervention, post-intervention and 6-month follow-up ( $n=89$ for CG; $n=117$ for $E G$ )

\begin{tabular}{|c|c|c|c|c|c|c|}
\hline & \multicolumn{2}{|c|}{ Pre-intervention } & \multicolumn{2}{|c|}{ Post-intervention } & \multicolumn{2}{|c|}{ Follow-up } \\
\hline & Mean & SE & Mean & SE & Mean & SE \\
\hline \multicolumn{7}{|c|}{$\begin{array}{l}\text { Mode of commuting } \\
\quad\left(n^{\circ} / \text { week }\right)^{a}\end{array}$} \\
\hline \multicolumn{7}{|c|}{ Walk } \\
\hline CG & 4.5 & 0.4 & 5.2 & 0.4 & 4.4 & 0.4 \\
\hline$E G$ & 4.4 & 0.3 & 4.7 & 0.3 & 5.5 & 0.3 \\
\hline$P$ (groups) & 0.884 & & 0.427 & & 0.067 & \\
\hline \multicolumn{7}{|l|}{ Bike } \\
\hline CG & 0.2 & 0.0 & 0.2 & 0.0 & 0.2 & 0.0 \\
\hline$E G$ & 0.1 & 0.0 & 0.0 & 0.1 & 0.1 & 0.0 \\
\hline$P$ (groups) & 0.889 & & 0.235 & & 0.085 & \\
\hline \multicolumn{7}{|l|}{ Car } \\
\hline$C G$ & 3.8 & 0.3 & 4.0 & 0.4 & 3.8 & 0.4 \\
\hline$E G$ & 4.0 & 0.4 & 3.3 & 0.4 & 3.1 & 0.3 \\
\hline$P$ (groups) & 0.778 & & 0.280 & & 0.242 & \\
\hline \multicolumn{7}{|l|}{ Motorcycle } \\
\hline$C G$ & 0.0 & 0.0 & 0.0 & 0.0 & 0.2 & 0.1 \\
\hline$E G$ & 0.1 & 0.0 & 0.0 & 0.0 & 0.2 & 0.1 \\
\hline$P$ (groups) & 0.513 & & 0.560 & & 0.787 & \\
\hline \multicolumn{7}{|l|}{ Bus } \\
\hline$C G$ & 0.5 & 0.2 & 0.6 & 0.2 & 0.6 & 0.2 \\
\hline$E G$ & 0.4 & 0.1 & 0.8 & 0.2 & 0.6 & 0.2 \\
\hline$P$ (groups) & 0.674 & & 0.498 & & 0.944 & \\
\hline
\end{tabular}

Data are shown as mean and standard error.

$E G$, experimental group.

a: Number of travels to and from school per week (range: 0-10). CG, control group;

One-way analysis of co-variance (dependent variable = frequency of the mode of commuting, fixed factor=group).

Descriptive values for $p$ values are adjusted by sex, age and distance.

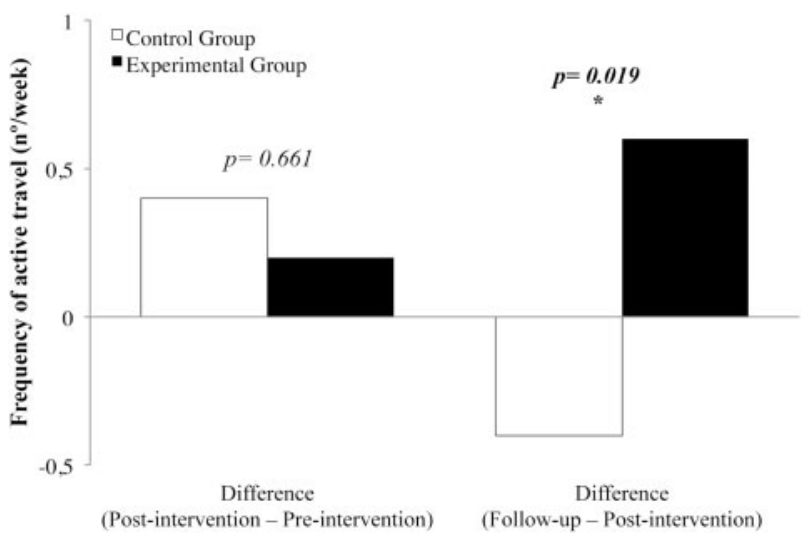

Figure 1 Change of the frequency of active commuting at postintervention and 6-month follow-up

different on the frequency of active commuting between groups at pre-intervention, post-intervention and 6-month follow-up. There was a statistically significant difference in the change of the mode of commuting to school between the control and experimental groups at 6-month follow-up (Follow-up-Post-intervention difference) but only for walking mode (number of walk travels per week: CG $=-0.6 \pm 0.3 ; \mathrm{EG}=0.7 \pm 0.2 ; p=0.004)$. However, there were no changes in the other modes of commuting: bike $(p=0.100)$, car $(p=0.566)$, motorcycle $(p=0.267)$ and bus $(p=0.407)$ (table 2$)$.

\section{Discussion}

We found significant differences in the change of the frequency of active commuting and mode (only walking) between the control and

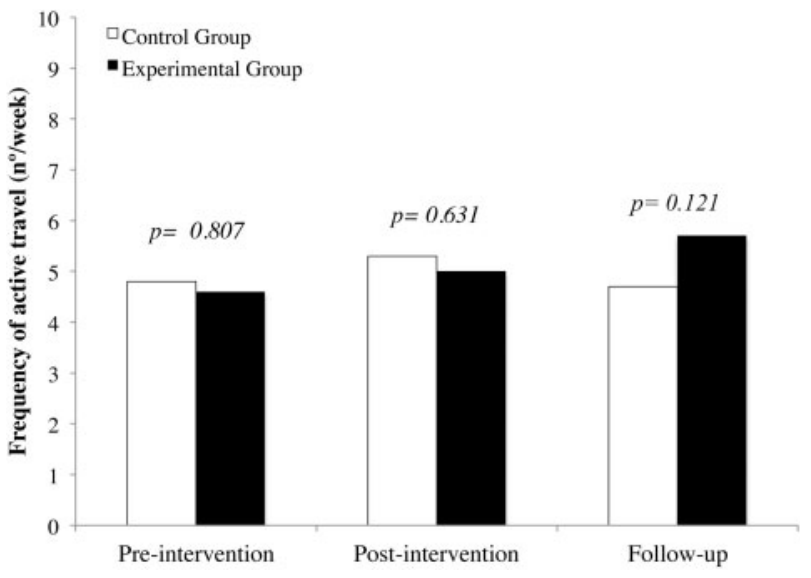

Figure 2 Descriptive values and $p$ values of the frequency of active commuting between the control and experimental groups at preintervention, post-intervention and follow-up

experimental groups at 6-month follow-up. However, we assume that this change 6 month after the end of the intervention period was low, since there was only an increase of 0.6 active travels/week and 0.7 walk travels/week.

As noted in a recent review of interventions to promote active school travel, all of the identified studies performed at least two measures of active commuting to school before (pre-intervention) and after (postintervention). ${ }^{13}$ However, few intervention studies investigated the maintenance of the intervention effect at follow-up. ${ }^{14,15}$

In the current study, we evaluated the frequency of active commuting to school 6 months after the end of the intervention period. To our knowledge, only two prior studies investigated the effects of the intervention on active commuting to school following the post-intervention measurement period. ${ }^{14,15}$ In the first study, American children participated in two designated days for walking and bicycling to school: the 'International Walk to School Day' in the fall and 'Fill the Racks!' in the spring. At both events, the students who walked and cycled were counted before the event day (the previous day), during the event day, and after the event day (the day after the event day). Further, in the 'Fill the Racks!' activity, active school travel of students was assessed 2 weeks later (follow-up). Both events showed a substantial increase on the frequency of walkers and cyclers at the event day and after the event day (post-intervention) compared with the day before the event (pre-intervention). In the spring event, 'Fill the Racks!' the data showed that the increased frequency on active commuting during the event day was sustained for at least 2 weeks after the event. ${ }^{14}$ However, this study reported only shortterm maintenance effects ( 1 day and 2 weeks). Using a 6-month follow-up, the current study found a significant difference in the frequency of active commuting and mode choice (only walking) between the control and experimental groups. However, there is a main difference between the American and the current study regarding the post-intervention effects. In the American study there was a positive effect on active commuting to school just after the intervention and in the current study there was a nonsignificant effect immediately following the intervention. We hypothesize that the differences between studies may be due to the duration and type of activities of the intervention, since the American study performed just an event day and the activity was walked to school, whereas the current study performed a 6-month intervention period and the activities were more diversed. Fully results regarding the short effects of the intervention programme are currently under review (submitted manuscript).

In the second maintenance study, Belgian children participated in an intervention based on cycle training, which included four sessions of 45 min during over a course of 4 -weeks. Parents were asked about 
Table 2 Change of the frequency of the mode of commuting at post-intervention and 6-month follow-up ( $n=89$ for CG; $n=117$ for $E G)$

\begin{tabular}{|c|c|c|c|c|}
\hline & \multicolumn{2}{|c|}{$\begin{array}{l}\text { Difference (post- } \\
\text { intervention- } \\
\text { pre-intervention) }^{\mathbf{b}}\end{array}$} & \multicolumn{2}{|c|}{$\begin{array}{l}\text { Difference (follow-up- } \\
\text { post-intervention) }\end{array}$} \\
\hline & Mean & SE & Mean & SE \\
\hline \multicolumn{5}{|c|}{$\begin{array}{l}\text { Mode of commuting } \\
\left(\mathrm{n}^{\circ} . / \text { week }\right)^{\mathrm{a}}\end{array}$} \\
\hline \multicolumn{5}{|c|}{ Walk } \\
\hline CG & 0.6 & 0.3 & -0.6 & 0.3 \\
\hline$E G$ & 0.2 & 0.2 & 0.7 & 0.2 \\
\hline$P$ (groups) & 0.322 & & 0.004 & \\
\hline \multicolumn{5}{|l|}{ Bike } \\
\hline$C G$ & -0.1 & 0.0 & 0.1 & 0.0 \\
\hline$E G$ & 0.0 & 0.0 & -0.0 & 0.0 \\
\hline$P$ (groups) & 0.101 & & 0.100 & \\
\hline \multicolumn{5}{|l|}{ Car } \\
\hline$C G$ & 0.1 & 0.3 & -0.0 & 0.3 \\
\hline$E G$ & -0.7 & 0.2 & -0.3 & 0.2 \\
\hline$P$ (groups) & 0.057 & & 0.566 & \\
\hline \multicolumn{5}{|l|}{ Motorcycle } \\
\hline$C G$ & -0.0 & 0.0 & 0.2 & 0.0 \\
\hline$E G$ & -0.0 & 0.0 & 0.1 & 0.0 \\
\hline$P$ (groups) & 0.860 & & 0.267 & \\
\hline \multicolumn{5}{|l|}{ Bus } \\
\hline$C G$ & 0.3 & 0.1 & -0.1 & 0.1 \\
\hline$E G$ & 0.1 & 0.1 & 0.0 & 0.1 \\
\hline$P$ (groups) & 0.574 & & 0.407 & \\
\hline
\end{tabular}

Data are shown as mean and standard error.

a:Number of travels to and from school per week (range: 0-10). CG, control group; EG, experimental group.

One-way analysis of co-variance (dependent variable $=$ postintervention-pre-intervention differences and follow-up-postintervention differences, fixed factor $=$ group).

b:Descriptive values for the differences and $p$ values are adjusted by sex, age, distance, attendance and the corresponding pre-intervention/post-intervention commuting variable.

their child's cycling behaviour to school (number of trips and duration) at pre-intervention, within 1 week after the last session (post-intervention) and at 5-months follow-up. No significant intervention effects were found on children's cycling frequency to school $(F=1.9)$ either short- or longer-term. ${ }^{15} \mathrm{~A}$ similar result was observed in the current study regarding the frequency of bicycling, however, it should be highlighted that the number of cyclists in our study was very low $(0.1 \%)$ and conclusions cannot be made based on this low number of participants. Belgium is a country with a higher cycling tradition than Spain, which includes not only the younger members but also the entire population. Consequently, comparisons must be done cautiously because of this environmental and contextual difference. Moreover, the intervention activities of both studies differed consistently. In the current study, a statistically significant difference was found only for walking mode, may be due to the targeting of the most of activities [except (iv)], which were mostly focused on walking but not on cycling. In the Belgium study, the primary aim was to evaluate the short- and longer-term effects of a cycle training on children's cycling skills and active commuting was a secondary objective. Thus, their findings are consistent with the conclusions of Chillón et al., ${ }^{13}$ who noted in a review of active commuting studies that interventions focused on active transportation to school (i.e. the first objective being to increase the number of walkers or cyclists) may be more effective than those with a broader focus (i.e. those where the first objective is not necessarily to increase the number of walkers or cyclists). On the other hand, both previous studies have used the same active commuting measure (i.e. questionnaire), as the current study.
The results of the current study only confirmed significant changes in the mode of commuting of walking. However, a change in a mode of commuting implies necessarily changes in other modes among the same sample, and these changes on the mode of commuting must be interrelated. In this study, the number of walkers increased and the number of car users decreased (although it was no significant), so we might confirm that both changes derived from the same cause (i.e. the intervention). The aim of the interventions to promote active commuting is to increase the rate of active commuting by reducing the rate of passive commuting or vice versa.

There is evidence regarding the positive association between the mode of commuting to work of parents and the mode of school travel of their children. ${ }^{22,23}$ Consequently, modifying the behaviour of the mode of commuting to school in the children might affect the mode of travel to work from the parents or vice versa. Since both children's and parent's decisions on how to get to school and the mode of travel might require a family planning strategy to address several issues (e.g. starting times, availability of vehicles in the family, distances from home to school/work, public transportation), the fact of changing the mode of commuting in a family (e.g. from passive to active) might require quite a long time to reorganize and it may occur at long-term. This might be a reasonable justification for understanding the positive long-term effect on the increasing the rate of active commuting to school 6 months after the intervention finished, but not immediately after the intervention period.

It is unknown how long a child needs to acquire and integrate a healthy behaviour, such as active commuting to school, in their daily routine. Further long-term maintenance studies are needed to determine whether the intervention effect on active commuting to school persists at long-term, or even increase or decrease after the intervention. Moreover, these studies will provide a more accurate summary of the key successful points of the interventions focused on changing behaviours to adopt healthier lifestyles, such as active commuting to school. ${ }^{24,25}$

Furthermore, three main issues should be addressed to encourage more children to use active commuting to school, including: (a) the parent's perceptions of the environment (i.e. perceptions of safety from home to school and back), ${ }^{26}$ (b) the school implementation of educational strategies within the curriculum ${ }^{27}$ and (c) the school implementation of environmental strategies (e.g. infrastructure such as set cycle parking at schools).

The present intervention study has some limitations. Usual physical activity behaviour was not assessed, although all students performed 2 weekly hours of Physical Education. Because this was a modest intervention (there was only one intervention activity each month), it is unclear whether a more intensive intervention could have a greater increasing effect. Allocation to control or experimental groups was non-random. Almost $60 \%$ of the participants at baseline were dropped in this study due to missing data. On the other hand, children were the most important target group, while parents participated only in one activity (i), and it would explain the limited intervention effects. Finally, information on participant's socio-economic status (SES) and other variables that might provide deeper information on the results (e.g. physical activity, familiar variables) were not known. A major strength of the study is the intervention design and the inclusion of a 6-month follow-up measurement. Future research should focus on how to assist children and their families to maintain an active commuting behaviour in a longer term and to investigate the time that children need to keep an active commuting behaviour in order to acquire it as a long-life habit.

As a conclusion, the present study showed that a 6-month schoolbased intervention focused on increasing the levels of active commuting to school might be effective on increasing the frequency of active commuting to school even beyond the period of intervention. 


\section{Acknowledgements}

The authors acknowledge the help of the participants that took part in the study and thank their parents for their collaboration. We are grateful to the team: Rafael Guillen, Sergio Castillo, Oscar Flores, Sebastian Montes, Milkana Borges, Alvaro J. García, Carlos González, Carlos Rodríguez and Vanesa Mirón who helped in the field tests and questionnaires in schools and they were undergraduate students from the University of Granada. This work is part of a Ph.D. Thesis conducted in the Biomedicine Doctoral Studies of the University of Granada, Spain.

Conflicts of interest: None declared.

\section{Key points}

- Intervention studies are needed to identify the most successful strategies for increasing the frequency of active commuting to school.

- A school-based intervention focused on active commuting to school might increase the frequency of active commuting at 6-month follow-up.

- Changing the mode of commuting in children is influenced by the overall family (i.e. parents, brothers ... ), and it might require longer times to reorganize the family planning.

- Educational campaigns to encourage active commuting should be focus on parents and children.

\section{References}

1 Janssen I, LeBlanc A. Systematic review of the health benefits of physical activity and fitness in school-aged children and youth. Int J Behav Nutr Phy 2010;7:40.

2 Faulkner GEJ, Buliung RN, Flora PK, Fusco C. Active school transport, physical activity levels and body weight of children and youth: a systematic review. Prev Med 2009;48:3-8.

3 Larouche R, Saunders TJ, Faulkner GEJ, et al. Associations between active school transport and physical activity, body composition, and cardiovascular fitness: a systematic review of 68 studies. J Phys Act Health 2014;11:206-27.

4 Martinez-Gomez D, Veiga OL, Gomez-Martinez S, et al Behavioural correlates of active commuting to school in Spanish adolescents: the AFINOS (physical activity as a preventive measure against overweight, obesity, infections, allergies, and cardiovascular disease risk factors in adolescents) study. Public Health Nutr 2011;14:1779-86.

5 Lambiase MJ, Barry HM, Roemmich JN. Effect of a simulated active commute to school on cardiovascular stress reactivity. Med Sci Sports Exerc 2010;42:1609-16.

6 Wilson EJ, Wilson R, Krizek KJ. The implications of school choice on travel behavior and environmental emissions. Transport Res D-Tr E 2007;12:506-18.

7 McDonald NC. Active transportation to school: trends among U.S. schoolchildren, 1969-2001. Am J Prev Med 2007;32:509-16.
8 Chillon P, Martinez-Gomez D, Ortega FB, et al Six-year trend in active commuting to school in Spanish adolescents: the AVENA and AFINOS studies. Int J Behav Med 2012;20:529-37.

9 McDonald NC, Steiner RL, Lee C, et al Impact of the safe routes to school program on walking and bicycling. J Am Plann Assoc 2014;80:153-67.

10 Yang Y, Diez-Roux A, Evenson KR, Colabianchi N. Examining the impact of the walking school bus with an agent-based model. Am J Public Health 2014;104: 1196-203.

11 Wen LM, Fry D, Rissel C, et al Factors associated with children being driven to school: implications for walk to school programs. Health Educ Res 2008;23:325-34.

12 Mammen G, Stone MR, Faulkner G, et al Active school travel: an evaluation of the Canadian school travel planning intervention. Prev Med 2014;60:55-9.

13 Chillón P, Evenson KR, Vaughn A, Ward DS. A systematic review of interventions for promoting active transportation to school. Int J Behav Nutr Phy 2011;8:10.

14 Buckley A, Lowry MB, Brown H, Barton B. Evaluating safe routes to school events that designate days for walking and bicycling. Transport Policy 2013;30:294-300.

15 Ducheyne F, De Bourdeaudhuij I, Lenoir M, Cardon G. Effects of a cycle training course on children's cycling skills and levels of cycling to school. Accid Anal Prev 2014;67:49-60.

16 Panter J, Jones A, van Sluijs E. Environmental determinants of active travel in youth: a review and framework for future research. Int J Behav Nutr Phy 2008;5:34

17 Bors $\mathrm{P}$, Dessauer M, Bell R, et al The active living by design national program community initiatives and lessons learned. Am J Prev Med 2009;37:S313-21.

18 McMillan TE. Urban form and a child's trip to school: the current literature and a framework for future research. J Planning Literature 2005;19:440-56.

19 Pikora T, Giles-Corti B, Bull F, et al Developing a framework for assessment of the environmental determinants of walking and cycling. Soc Sci Med 2003;56:1693-703.

20 Herrador-Colmenero M, Pérez-García M, Ruiz JR, Chillón P. Assessing modes and frequency of commuting to school in youngsters: a systematic review. Pediatr Exerc Sci 2014;26:291-341.

21 Mendoza JA, Watson K, Baranowski T, et al Ethnic minority children's active commuting to school and association with physical activity and pedestrian safety behaviors. J Appl Res Child 2010;1:1-23.

22 Henne HM, Tandon PS, Frank LD, Saelens BE. Parental factors in children's active transport to school. Public Health 2014;128:643-6.

23 Rodriguez-Lopez C, Villa-Gonzalez E, Perez-Lopez IJ, et al Family factors influence active commuting to school in Spanish children. Nutr Hosp 2013;28:756-63.

24 Jepson RG, Harris FM, Platt S, Tannahill C. The effectiveness of interventions to change six health behaviours: a review of reviews. BMC Public Health 2010;10:538.

25 Marcus BH, Dubbert PM, Forsyth LH, et al Physical activity behavior change: issues in adoption and maintenance. Health Psychol 2000;19:32-41.

26 Chillon P, Hales D, Vaughn A, et al A cross-sectional study of demographic, environmental and parental barriers to active school travel among children in the United States. Int J Behav Nutr Phy 2014;11:61.

27 Jordan KC, Erickson ED, Cox R, et al Evaluation of the gold medal schools program. J Am Diet Assoc 2008;108:1916-20. 